\title{
ARTICLE OPEN \\ Comparative integrated omics: identification of key functionalities in microbial community-wide metabolic networks
}

\author{
Hugo Roume ${ }^{1,5,6}$, Anna Heintz-Buschart ${ }^{1,6}$, Emilie EL Muller ${ }^{1}$, Patrick May ${ }^{1}$, Venkata P Satagopam ${ }^{1}$, Cédric C Laczny ${ }^{1}$, \\ Shaman Narayanasamy ${ }^{1}$, Laura A Lebrun ${ }^{1}$, Michael R Hoopmann ${ }^{2}$, James M Schupp ${ }^{3}$, John D Gillece ${ }^{3}$, Nathan D Hicks ${ }^{3}$, \\ David M Engelthaler ${ }^{3}$, Thomas Sauter ${ }^{4}$, Paul S Keim ${ }^{3}$, Robert L Moritz ${ }^{2}$ and Paul Wilmes ${ }^{1}$
}

BACKGROUND: Mixed microbial communities underpin important biotechnological processes such as biological wastewater treatment (BWWT). A detailed knowledge of community structure and function relationships is essential for ultimately driving these systems towards desired outcomes, e.g., the enrichment in organisms capable of accumulating valuable resources during BWWT. METHODS: A comparative integrated omic analysis including metagenomics, metatranscriptomics and metaproteomics was carried out to elucidate functional differences between seasonally distinct oleaginous mixed microbial communities (OMMCs) sampled from an anoxic BWWT tank. A computational framework for the reconstruction of community-wide metabolic networks from multi-omic data was developed. These provide an overview of the functional capabilities by incorporating gene copy, transcript and protein abundances. To identify functional genes, which have a disproportionately important role in community function, we define a high relative gene expression and a high betweenness centrality relative to node degree as gene-centric and network topological features, respectively.

RESULTS: Genes exhibiting high expression relative to gene copy abundance include genes involved in glycerolipid metabolism, particularly triacylglycerol lipase, encoded by known lipid accumulating populations, e.g., Candidatus Microthrix parvicella. Genes with a high relative gene expression and topologically important positions in the network include genes involved in nitrogen metabolism and fatty acid biosynthesis, encoded by Nitrosomonas spp. and Rhodococcus spp. Such genes may be regarded as 'keystone genes' as they are likely to be encoded by keystone species.

CONCLUSION: The linking of key functionalities to community members through integrated omics opens up exciting possibilities for devising prediction and control strategies for microbial communities in the future.

npj Biofilms and Microbiomes (2015) 1, 15007; doi:10.1038/npjbiofilms.2015.7; published online 17 June 2015

\section{INTRODUCTION}

Our ability to study microbial communities in natural settings as well as in engineered systems, e.g., biological wastewater treatment (BWWT) plants, has dramatically improved in recent years owing to rapid advances in high-throughput DNA sequencing technologies and other 'meta-omic' analyses which are driving molecular microbial ecology into the era of Eco-Systems Biology. ${ }^{1}$ Although metagenomic data provide gene inventories, without any proof of their functionality, the analysis of community-wide transcripts facilitates an assessment of community-wide functions, ${ }^{2}$ and community proteomics provide representation of the actual phenotypic traits of individual community members. ${ }^{3}$ Metabolomics, through resolving the final and intermediate products of cellular metabolism, should theoretically be the most sensitive indicator of community-wide phenotypes and allow inference of key metabolic processes. ${ }^{4}$ However, current metabolomic methodologies are limited in the number of metabolites that can be measured as well as their limited identifiability. ${ }^{5}$
The reconstruction of metabolic networks based on genomic data presents a compelling alternative to metabolomics for resolving the metabolic capabilities of organisms. ${ }^{6}$ So far, the conventional approach used to progress from single to multispecies metabolic network reconstructions has involved treating the metabolic networks of individual species as an input-output system to build network-based ${ }^{7}$ or constraint-based ${ }^{8}$ models of metabolic interactions. However, these multi-species models, which are usually limited to only a few species, fail to explain how variations in gene or species composition affect the overall metabolic state of ecosystems. ${ }^{9}$ Given the complexity of microbial communities, as well as the inability to isolate and sequence representative single cultures of all organisms within a community, such bottom-up approaches may be limited by the inherent impossibility to extrapolate community-wide networks and behaviour from individual isolate omic data sets. ${ }^{1}$ Recently developed alternative approaches involve the determination of community-wide metabolic potential ${ }^{10}$ and the reconstruction of community-wide metabolic networks based directly on

\footnotetext{
${ }^{1}$ Luxembourg Centre for Systems Biomedicine, University of Luxembourg, Esch-sur-Alzette, Luxembourg; ${ }^{2}$ Institute for Systems Biology, Seattle, WA, USA; ${ }^{3}$ The Translational Genomic Research Institute-North, Flagstaff, AZ, USA and ${ }^{4}$ Life Science Research Unit, University of Luxembourg, Luxembourg, Luxembourg.

Correspondence: P Wilmes (paul.wilmes@uni.lu)

${ }^{5}$ Current address: Laboratory of Microbial Ecology and Technology, Ghent University, Ghent, Belgium

${ }^{6}$ These authors contributed equally to this work.
}

Received 16 February 2015; revised 24 April 2015; accepted 6 May 2015 
a

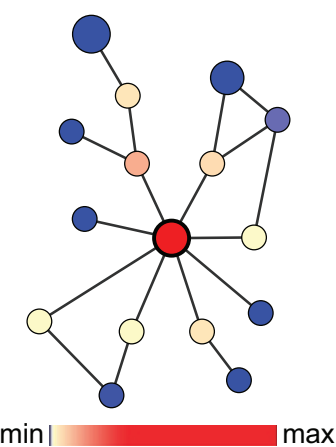

Topological features:

- High node degree

- High betweenness centrality

Node attribute:

- High impact activity b

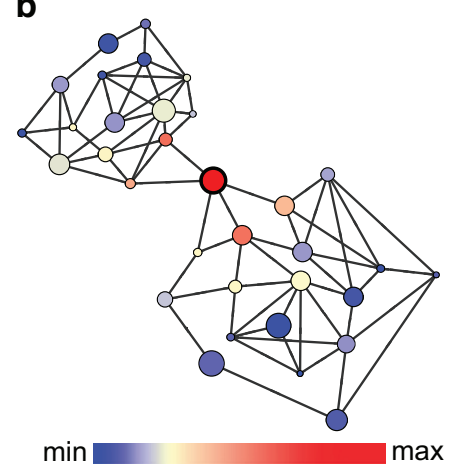

Topological features:

- Low relative degree

- High betweenness centrality

Node attribute:

- High relative expression

Figure 1. Criteria for defining keystone nodes in microbial species interaction and community-wide metabolic networks. (a) Criteria for identifying keystone species in reconstructed species interaction networks. Nodes represent taxa and edges represent associations between them. Node sizes reflect activity. (b) Criteria for identifying genes encoding key functionalities in reconstructed communitywide metabolic networks. Nodes represent enzyme-coding genes and edges correspond to shared metabolites (either reactants, products or educts). Node sizes reflect relative expression.

metagenomic data, ${ }^{11}$ thereby ignoring the contribution of individual species. ${ }^{12}$ Through this population-independent approach, Greenblum et al. ${ }^{12}$ identified enzyme-coding genes, either enriched or depleted, in stool samples of human individuals with obesity or inflammatory bowel disease, highlighting the potential of such approaches for the identification of key metabolic traits within microbial consortia. Ideally, top-down and bottom-up approaches should be combined to identify links between microbial community structure and function, thereby bridging the gap between population-level metabolic networks and the larger community-wide networks to ultimately build a systems-level model of interactions between species. ${ }^{13}$

Here, we discuss a framework for comparative integrated omic analyses, which allows integration of systematically generated multi-omic data within reconstructed community-level metabolic networks. The resulting networks allow assessment of gene expression and protein abundances in combination with network topological features. We propose the use of these networks as an alternative to identifying keystone species through co-occurrence networks ${ }^{14}$ (Figure 1a). Reconstruction of co-occurrence networks requires large numbers of highly resolved samples and spurious correlations can affect interpretability of the resulting networks. ${ }^{15}$ Here, we identify genes encoding key functionalities in reconstructed community-wide metabolic networks and trace these back to the community members which encode them. Through their activity, keystone species are expected to have a disproportionately large effect on their environment, relative to their abundance. $^{16}$ Their removal would greatly impact community structure and function. ${ }^{17}$ For example, in the human colon, specialist primary degraders such as Ruminococcus bromii are considered keystone species because of their ability to initiate the degradation of recalcitrant substrates. ${ }^{18}$ Herein, we define key functionalities as specific functions which have an overall pronounced effect on ecosystem functioning, because they exhibit a high relative gene expression and are represented by a

node with a prominent topological position within a communitywide metabolic network (Figure 1b). The loss of such nodes would result in a lack of connectivity and this would greatly impact the overall topology of the community-wide metabolic network. In addition, the expression of these genes will likely be rate-limiting, similar to the effect of 'load points' on reconstructed singleorganism metabolic networks, ${ }^{19}$ and thereby will govern the metabolic outcomes of the entire community. Therefore, by altering the expression of such genes, the community-wide phenotype could be influenced. By extension, members of the microbial community carrying out these functions would likely also be keystone species.

We apply the developed methodological framework to oleaginous mixed microbial communities (OMMCs) sampled from the surface of an anoxic BWWT tank in autumn and winter, respectively (Figure 2a,b). BWWT plants exhibit well-defined physical boundaries and represent a convenient and virtually unlimited source of spatially and temporally resolved samples. The microbial communities found in BWWT plants represent an ideal model system for microbial ecology ${ }^{20}$ because these communities are comparatively well described and lie between communities of low diversity, e.g., acid mine drainage biofilms, ${ }^{21}$ and complex communities such as those found in the human gastrointestinal tract $^{22}$ or soil environments ${ }^{23}$ while retaining important hallmarks of both ends of the spectrum. These characteristics include (i) levels of dominance of individual taxa typically associated with low diversity communities (up to $30 \%$ of the community), most notably either Candidatus Microthrix parvicella (henceforth referred to as Microthrix parvicella) or Perlucidibaca spp. depending on the time of year; ${ }^{24}$ and (ii) the functional potential to adapt to rapid environmental changes typically observed in more diverse communities. Compared with BWWT microbial communities that are more typically studied, e.g., bulk activated sludge, OMMCs have additional important attributes which render them ideally suited as a model for the development and implementation of eco-systematic approaches. These include (i) limited species richness, i.e., operational taxonomic unit (OTU) richness of approximately 600 (Chao $^{25}$ estimate from previous data ${ }^{24}$ ) compared with more than 1,000 (ref. 26) for activated sludge; (ii) high reproducibility between samples taken at the same time point. ${ }^{4,27}$ Apart from these characteristics, the targeted enrichment of OMMCs is of biotechnological interest as this would allow the reclamation of a significant fraction of the chemical energy contained within wastewater through lipid recovery and subsequent biodiesel synthesis. ${ }^{28,29}$ However, for such enrichment strategies to be successful, a detailed understanding of community function is necessary. ${ }^{30}$ For example, identified key functionalities may ultimately serve as driver nodes ${ }^{31}$ for controlling these communities.

\section{MATERIALS AND METHODS}

\section{Sampling}

OMMCs were sampled from the anoxic tank of the Schifflange (Esch-surAlzette, Luxembourg; $49^{\circ} 30^{\prime} 48.29^{\prime \prime} \mathrm{N} ; 6^{\circ} 1^{\prime} 4.53^{\prime \prime} \mathrm{E}$ ) BWWT plant as described previously. ${ }^{4}$ Samples were taken on 4 October 2010 (referred to herein as the autumn OMMC) and 25 January 2011 (referred to herein as the winter OMMC; physico-chemical characteristics of the wastewater on the sampling dates are provided in Supplementary Table 1). These dates were chosen because they are representative of both extremes of OMMC-wide phenotypes, whereby, during the autumn sampling date, the tank exhibited only sparse amounts of OMMC biomass (Figure 2a) and, on the winter sampling date, ample amounts of OMMC biomass were present (Figure 2b).

\section{Biomolecular extractions}

A previously developed biomolecular isolation framework for communityintegrated omics ${ }^{4,27}$ was used to sequentially extract total RNA, genomic 
a

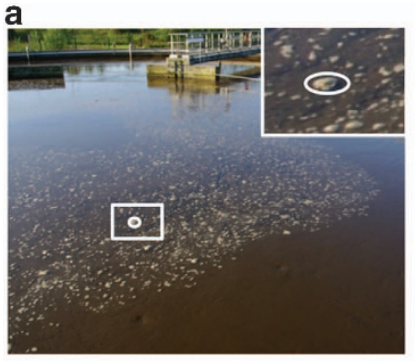

b

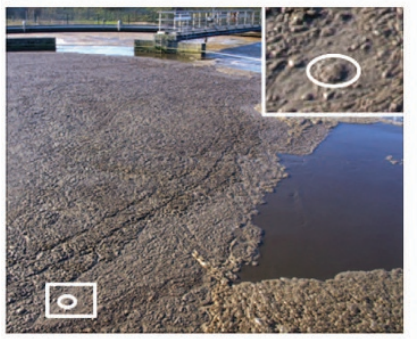

c

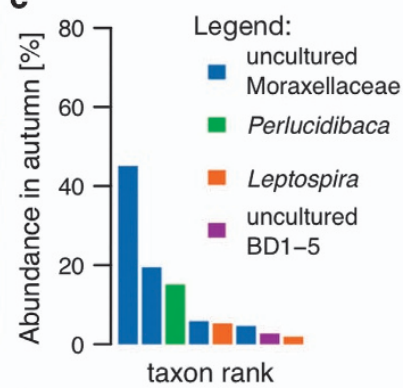

d

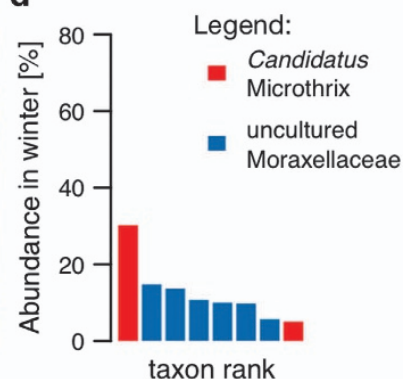

Figure 2. OMMC composition in autumn and winter seasons. Photographs of the OMMCs located at the water surface of the anoxic tank at the Schifflange BWWT plant in (a) autumn and (b) winter sampling dates. Abundance of genera of dominant community members based on reconstructed 16S rRNA gene sequences from metagenomic data in (c) autumn and (d) winter. OMMC, oleaginous mixed microbial community; rRNA, ribosomal RNA.

DNA and proteins from single OMMCs based on the Qiagen AllPrep DNA/RNA/Protein Mini kit (QA, Qiagen, Venlo, The Netherlands). The quality and quantity of isolated biomacromolecules were assessed as described previously ${ }^{4}$ (Supplementary Table 2, Supplementary Materials and methods).

\section{High-throughput sequencing}

Total genomic DNA and ribosomal RNA-depleted retrotranscribed CDNA from both samples were sequenced on an Illumina Genome Analyzer IIx (Supplementary Materials and methods). Raw metagenomic and metatranscriptomic sequence data files are accessible in nucleic acid databases under BioProject PRJNA230567, sample LAO-A01 (SRX612782 and SRX612783) and LAO-A02 (SRX389533 and SRX389534).

Metagenomic and metatranscriptomic sequence assembly, gene annotation and determination of gene abundances

Raw $100 \mathrm{nt}$ paired-end sequencing reads from the metagenome and metatranscriptome libraries from each of the two sampling dates were first trimmed and quality filtered using the trim-fasta.pl script from the PoPoolation package ${ }^{32}$ and overlapping read pairs were assembled using the PAired-eND Assembler ${ }^{33}$ (PANDAseq). Non-redundant assembled PANDAseq read pairs and non-assembled reads from metagenomic and metatranscriptomic data sets of both sampling dates were then used as a single input for the MOCAT assembly pipeline. ${ }^{34}$ The resulting nonredundant contigs and PANDAseq-assembled read pairs that had not been used were then combined and filtered with a minimum length threshold of $150 \mathrm{bp}$. Protein-coding genes were predicted using the Prodigal gene finder ${ }^{35}$ (v2.60, contigs above $500 \mathrm{bp}$ ) or FragGeneScan ${ }^{36}$ (contigs between 150 and $500 \mathrm{bp}$ ). The resulting amino acid sequences from both contig sets were merged and made non-redundant using CD-HIT. ${ }^{37}$ All predicted gene sequences are accessible through MG-RAST $T^{38}$ as ID MGM4550606.3. The Kyoto Encyclopedia of Genes and Genome ${ }^{39}$ database version 64.0 was used to functionally annotate genes with Kyoto Encyclopedia of Genes and Genome orthologous groups (KOs) for ensuing metabolic network reconstruction (Supplementary Materials and methods, Supplementary Figure 1).

To allow meaningful comparisons between gene copy and transcript numbers from the two seasons, identical numbers of reads were sampled from the metagenomic and the metatranscriptomic libraries of both seasons (Supplementary Materials and methods) using an in-house developed Perl-script. The resulting reads were then mapped to the annotated gene sets. Cross-mapping reads were equally weighted according to the number of genes they mapped to and mapped reads were counted per gene. Finally, metagenomic and metatranscriptomic counts were normalised by the effective length of the gene sequence ${ }^{40}$ yielding normalised gene copy abundances and normalised transcript abundances, respectively. KO abundances were inferred from the sums of normalised gene copy or transcript abundances of all genes belonging to a given KO (Supplementary Materials and methods). Relative gene expression values were determined per KO by calculating the ratio of normalised transcript abundances to normalised gene copy abundances (Supplementary Materials and methods, Supplementary Dataset 3).

\section{Metaproteome processing and analysis}

Isolated and purified protein fractions were separated using onedimensional SDS polyacrylamide gel electrophoresis. The proteins were reduced, alkylated, and digested with trypsin. The resulting peptides were then analysed by liquid chromatography coupled to tandem mass spectrometry. Peptide identification was carried out by database searching using the $X$ !Tandem software ${ }^{41}$ with the amino acid sequence database generated from the genes predicted from the combined metagenomic and metatranscriptomic assembly. Protein identification was carried out using peptide-spectrum matches using the Trans-Proteomic Pipeline, ${ }^{42}$ with a probability of being correctly assigned to each protein determined by PeptideProphet. ${ }^{43}$ The protein inferences from each fraction were determined using ProteinProphet and then combined with iProphet ${ }^{44}$ to obtain a master set of identified proteins at a $1 \%$ false discovery rate. All proteomic data have been deposited in the PeptideAtlas mass spectrometry raw file repository at http://www.peptideatlas.org/PASS/PASS00512. Identified proteins were assigned KO numbers using BLAT-based ${ }^{45}$ alignment against the Kyoto Encyclopedia of Genes and Genome database v64.0 (Supplementary Materials and methods). Relative protein abundances were obtained using the normalised spectral index, as described previously $^{24}$ (Supplementary Materials and methods, Supplementary Figure 4).

\section{Community-wide metabolic network reconstructions}

Community-wide metabolic networks were reconstructed from the KOs with metabolic functions identified in the predicted gene sets from the combined metagenomic and metatranscriptomic assembly. The network reconstructions were rendered season-specific by using only KOs with mapped metatranscriptomic reads from each of the two sampling dates. The reconstructed networks reflect a connectivity-centred view of metabolism whereby enzymes grouped by KOs are represented by nodes and metabolites are represented by undirected edges, which represent either substrate or products of reactions catalysed by the respective KOs. ${ }^{12}$ Each $\mathrm{KO}$ was assigned a pair-set of substrate and product metabolites according to the RPAIR ${ }^{46}$ annotation in Kyoto Encyclopedia of Genes and Genome database version 67.1 (Supplementary Materials and methods).

\section{Topological network analysis and selection criteria for genes} encoding key functionalities

To carry out a topological analysis of the reconstructed metabolic network, nodes and edges were rendered non-redundant, by representing multiple $\mathrm{KO}$ with identical substrate and product metabolites as a single node. A comparison between the non-redundant network and a redundant version was also carried out (Supplementary Materials and methods). As most of the nodes that regroup several KOs represent subunits of the same enzyme, the small changes incurred on betweenness centrality and load by making the nodes non-redundant enhance the ability of these topological measures to identify key enzymes in the reconstructed community-wide metabolic networks (see also Supplementary Results and Discussion). Key functionalities were identified on the basis of topological criteria and relative gene expression. The topological selection criterion was defined in analogy to 'load points' as defined by Rahman and Schomburg ${ }^{19}$ in the context of reconstructed single-cell metabolic networks. Load points have the highest ratio of betweenness centrality (the number of valid shortest paths passing through them) relative to node degree (the number of neighbouring nodes; referred to as 'neighbourhood 
connectivity' by Rahman and Schomburg ${ }^{19}$ ). Node degree and betweenness centrality, among other topological measures, of each node were computed using the Cytoscape Network-Analyzer plug-in, ${ }^{47}$ considering the reconstructed network as undirected. These parameters were used to calculate load scores as defined in Equation (1).

$$
\text { load score } \text { lo }=\frac{\sum_{s \neq n \neq t}\left(\sigma_{s t}(n) / \sigma_{s t}\right)}{\sum k_{n} e}
$$

where $s$ and $t$ are nodes in the network different from $n, \sigma_{s t}$ is the number of shortest paths from $s$ to $t$, and $\sigma_{s t}(n)$ is the number of shortest paths from $s$ to $t$ that $n$ lies on, $k_{n}$ denotes the node degree of $n$, and $\Sigma e$ denotes the total number of edges in the network. Thus, load score describes the number of reaction paths or conversions between metabolites that utilise a given enzyme, relative to its connectivity. It therefore serves as a proxy for an enzyme's contribution to the metabolic fluxes of the overall community.

We prioritised the nodes with the top 10 per cent of load scores. In addition to this topological criterion, the relative gene expression of a node (either from a single $\mathrm{KO}$ or nodes regrouping several KOs) was also taken into account, such that only $\mathrm{KO}$ with a high relative expression (top 10 per cent) were regarded as genes encoding key functionalities (Supplementary Materials and methods). Key functionalities were analysed for their involvement in the metabolism of uniquely occurring metabolites, i.e., to assess whether they represent 'choke points' as defined by Rahman and Schomburg. ${ }^{19}$ For the calculation of an alternative load score weighted according to the occurrence of the metabolites which should restrict 'load points' to nodes within pathways ${ }^{46}$ and a detailed analysis of sensitivity to the chosen cut-offs, see Supplementary Materials and methods.

Linking genes encoding key functionalities to specific organisms The presence of the identified genes in genomes of bacterial isolates was determined by aligning contigs bearing these genes to the contigs from genome assemblies of these strains using BLAST (Supplementary Materials and methods).

Isolate strain culture and whole-genome sequencing

OMMC biomass sampled on 12 October 2011 was cultured on different growth media recommended for the culture of bacteria from water and wastewater and isolation procedures followed (Supplementary Materials and methods). In all, 140 pure bacterial cultures were obtained and screened for lipid inclusions using the Nile Red fluorescent dye. ${ }^{48}$ Following DNA extraction using the Power Soil DNA isolation kit (MO BIO, Carlsbad, CA, USA), the genomes of 85 Nile Red-positive isolates were sequenced on an Illumina HiSeq Genome Analyzer IIx using the same sequencing approach as described for the metagenomic samples. The resulting sequencing reads were assembled using either the $A b y s s^{49}$ or the SPAdes ${ }^{50}$ assemblers (Supplementary Materials and methods). Based on the presence of a gene encoding a key functionality, one isolate (Isolate LCSB065) was selected for refinement of genome assembly as well as phylogenetic and genomic analysis (Supplementary Materials and methods).

Code availability and computational resources

All in-house developed scripts are available from the authors upon request. In silico analysis results were obtained using the high performance computing facilities of the University of Luxembourg. ${ }^{51}$

\section{RESULTS AND DISCUSSION}

Identification of functions encoded and expressed in OMMCs in autumn and winter

High-resolution coupled metagenomic, metatranscriptomic and metaproteomic data were generated from the OMMCs sampled in autumn and winter. A total of 16.2 gigabases $(\mathrm{Gb})$ of shotgun metagenomic paired-end $100 \mathrm{nt}$ read sequence data as well as $38.6 \mathrm{~Gb}$ of metatranscriptomic sequence data were obtained. 6.5 million genes were predicted from a 6.7 million contigs of a combined assembly ( $1.6 \mathrm{~Gb}$ total length) of all metagenomic and metatranscriptomic reads (Supplementary Table 3 ). Based on reconstructed $16 \mathrm{~S}$ ribosomal RNA gene sequences from the metagenomic data (Supplementary Materials and methods), the autumn and winter communities are dominated by Perlucidibaca spp. and Microthrix spp., respectively (Figure 2c,d, Supplementary Dataset 1). A total 830,679 predicted genes were annotated with KOs and regrouped (Materials and methods), yielding a total of 7,270 unique KOs. In the autumn sample, 10,074 protein groups (identified proteins grouped together because they share detected peptides) were identified using 19,248 non-redundant peptides out of a total of 727,155 mass spectra. In the winter sample, 7,106 protein groups were identified from 15,966 nonredundant peptides out of a total of 620,488 tandem mass spectra. A total 4,906 and 5,007 proteins were unambiguously identified in the autumn and winter samples, respectively.

The congruency between the metagenomic and metatranscriptomic data was high, as $92 \%$ of $\mathrm{KOs}$ represented in the metagenomic data are also present in the metatranscriptomic data for both autumn and winter data sets (Supplementary Dataset 2). The coverage of KOs was lower in the proteomic data, as $1,357 \mathrm{KOs}$ ( $26 \%$ of KOs annotated in the metagenomic data set) and $1,236 \mathrm{KOs}(23 \%)$ were identified in autumn and winter OMMCs, respectively. These proportions were mirrored by KOs within metabolic pathways (Figure 3a,b). This comparatively low metaproteomic coverage is due to current limitations in proteomic technologies for metaproteomic analyses. ${ }^{52}$

\section{Analysis of highly expressed genes in winter and autumn} communities

Given the limited depth of coverage in the proteomic data, we mainly focused our subsequent comparative analyses on the metagenomic and metatranscriptomic data. Metaproteomic results were, however, used to corroborate and validate interpretations based on the analysis of the metatranscriptomic data. The comparison of KOs present in the metagenomic and metatranscriptomic data sets highlighted 757 (12\%) and 210 (4\%) unique KOs in autumn and winter OMMCs, respectively. Similar results were found in the comparison of $\mathrm{KOs}$ from metabolic pathways (Figure $3 c$ ). This analysis highlights a relatively limited difference in terms of genetic potential and gene expression between the two seasonally distinct OMMCs despite stark differences in community structure (Figure 2c,d).

For each identified $\mathrm{KO}$, we calculated relative gene expression, which is considered to be more informative than simple transcript abundance because expression levels are normalised to metagenomic gene copy numbers. ${ }^{53}$ Furthermore, it allows quantitative insights into the contribution of low abundance members (such populations may be potential keystone species) to overall community activity to be obtained. ${ }^{54} \mathrm{KOs}$ with high relative expression in both seasons (Figure 3d,e, Supplementary Dataset 3) were further analysed, as these are good candidates for genes which likely affect the overall community phenotype. Among these, enrichments were found in KOs linked to nitrogen metabolism, as well as oxidative phosphorylation and nonribosomal peptide synthesis in both seasons (Supplementary Dataset 3). The highly expressed $\mathrm{KOs}$ involved in nitrogen metabolism represent enzymes for ammonium assimilation and oxidation, denitrification and nitrification. In particular, they include genes encoding likely subunits of ammonia monooxygenase (AMO; K10944, K10945 and K10946). AMO has a key role in the first step of nitrification carried out by aerobic ammonia-oxidising bacteria, mainly belonging to Nitrosomonas spp. and Nitrosospira spp. ${ }^{54}$ AMO was previously found to be highly expressed in BWWT biomass. ${ }^{55}$ In addition to the nitrogen metabolism enzymes expressed at a high level in both seasons, a nitrite reductase gene (K00363) was highly expressed in the autumn sample.

In the winter sample, the glycerolipid metabolism was enriched within highly expressed KOs. In particular, triacylglycerol lipase 
a

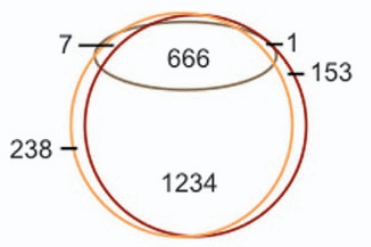

b

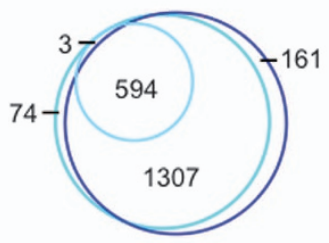

C

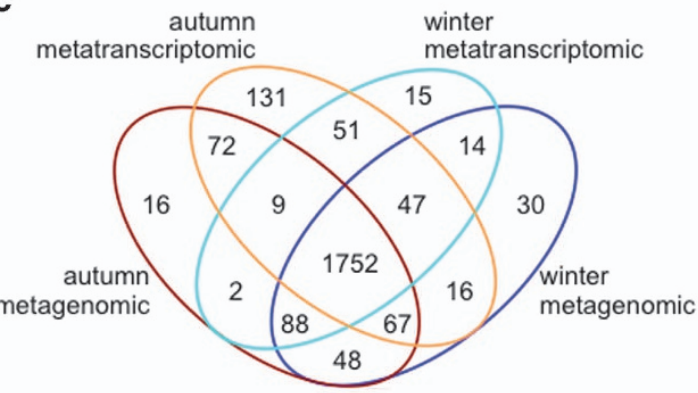

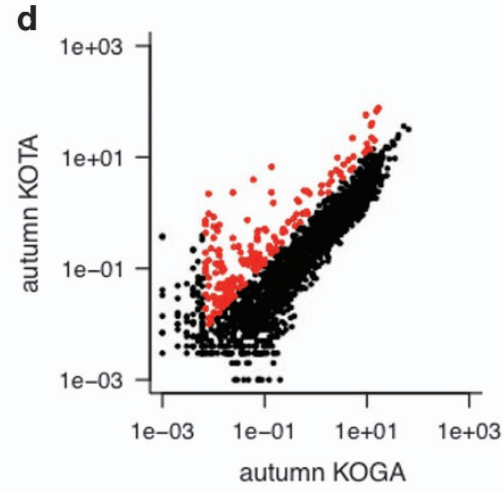

f

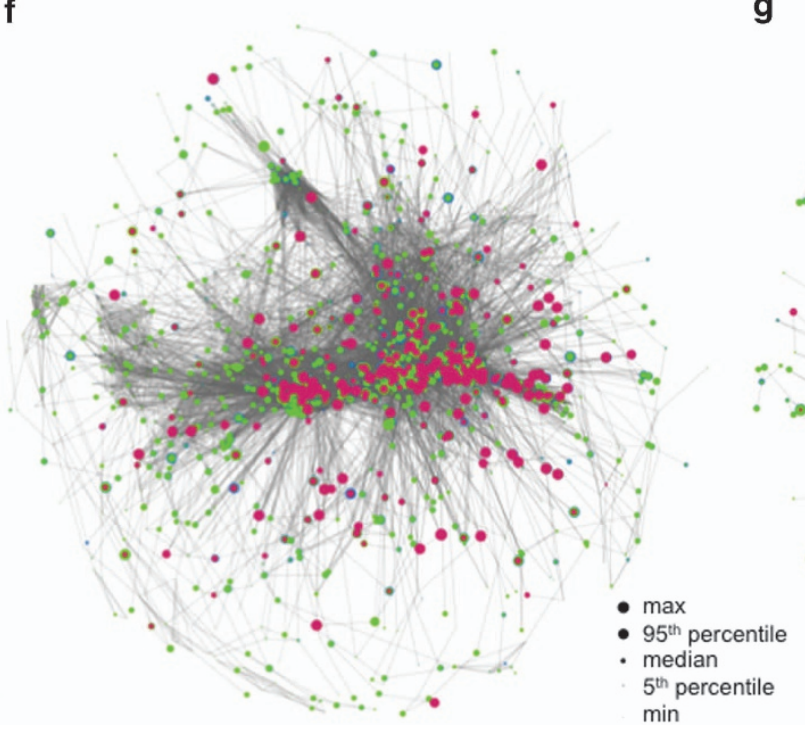

g e

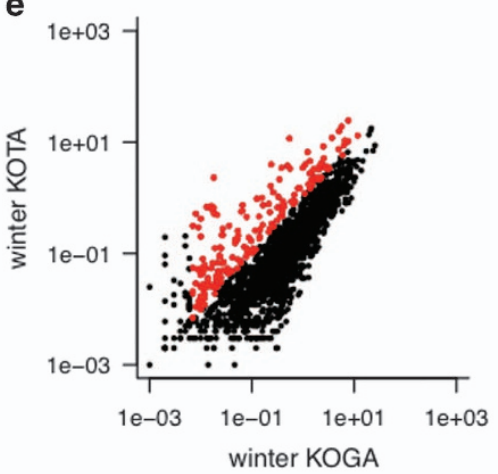

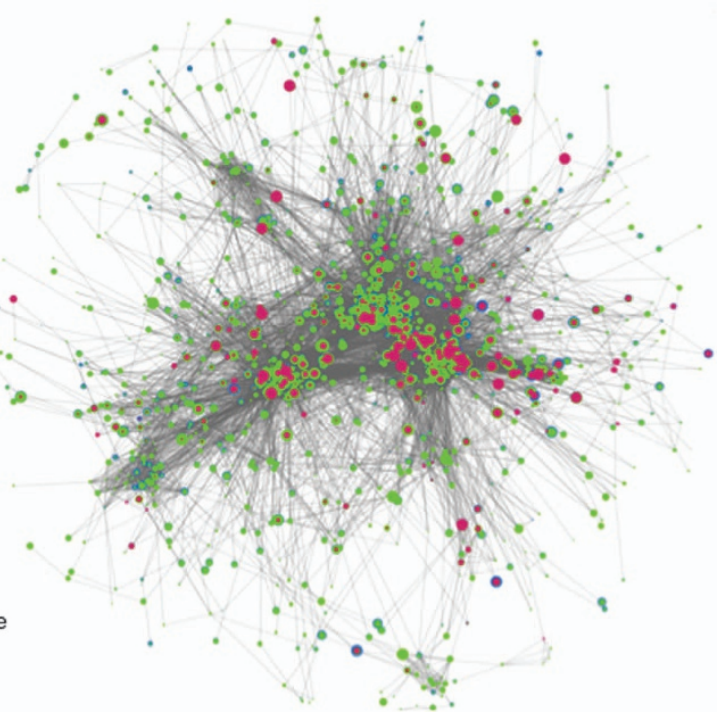

Figure 3. Integration of metagenomic, metatranscriptomic and metaproteomic data. (a) Venn diagram highlighting subsets of KEGG orthologous groups (KOs) in metabolic pathways present in the metagenomic (dark brown), metatranscriptomic (orange) and metaproteomic (pale brown) data from the autumn sample. (b) Subsets of KOs in metabolic pathways present in the metagenomic (dark blue), metatranscriptomic (cyan) and metaproteomic (pale blue) data from the winter sample. (c) Comparison of occurrence of KOs in metabolic pathways in metagenomic and metatranscriptomic data sets from autumn and winter. (d) Comparison of KO gene copy abundance (KOGA) and transcript abundance (KOTA) of KOs in metabolic pathways in the autumn data set. (e) Comparison of KO gene copy abundance (KOGA) and transcript abundance (KOTA) in metabolic pathways in the winter data set. In $\mathbf{d}$ and e, highly expressed KOs are highlighted in red. (f) Simplified autumn-specific metabolic network reconstruction. (g) Simplified winter-specific metabolic network reconstruction. In $\mathbf{f}$ and $\mathbf{g}$, size of nodes represents KO abundance at metagenomic (blue), metatranscriptomic (green) and metaproteomic (magenta) levels, respectively. KEGG, Kyoto Encyclopedia of Genes and Genome.

(K01046) exhibited pronounced transcript levels and its expression was also confirmed at the protein level (Supplementary Dataset 2). The most highly expressed genes of the 6,222 genes belonging to this $\mathrm{KO}$ could be matched to Acinetobacter spp., which are known to occur in BWWT plants and accumulate triacylglycerols. ${ }^{56}$ Furthermore, out of the genes with detectable expression, the two gene sequences with the highest gene copy numbers (i.e., abundance in the metagenomic data) were matched to the genome sequence of Microthrix parvicella BIO17-1 (ref. 57), which is enriched in KOs involved in lipid metabolism ${ }^{57}$ (11.3\% of its annotated genes). The presence of these enzymes was recently suggested to be essential for lipid accumulation in a metabolic 
model reconstruction of Microthrix parvicella, ${ }^{58}$ but not until now were they found to be expressed in biological wastewater treatment communities. The pronounced expression of the aforementioned KOs involved in ammonium oxidation and the hydrolysis of triacylglycerols during both seasons emphasises the capability of the OMMCs to remove two of the main compounds present in wastewater, i.e., ammonia ${ }^{59}$ and lipids. ${ }^{60}$

In the winter sample, KOs from the TCA cycle were also strongly expressed and the majority could be detected at the proteome level. Rather surprisingly, in the autumn sample, photosynthesis KOs were enriched. Expression of photosystem I in autumn was also confirmed by proteomics suggesting that phototrophic organisms are part of the floating OMMC during this season.

Reconstruction of a generalised and season-specific OMMC-wide metabolic networks

A community-wide metabolic network was reconstructed using the KOs expressed in the autumn and winter samples (Materials and methods, Supplementary Figure 5, Supplementary Dataset 4). The reconstructed network comprised 1,432 KO nodes with 29,988 edges representing non-unique metabolites.

Season-specific networks were reconstructed analogous to the generalised OMMC-wide network, but by only using the 1,885 KOs or $1,775 \mathrm{KOs}$ expressed in autumn or winter, respectively (Figure 3f,g, Supplementary Datasets 5 and 6). This yielded networks comprising 1,298 nodes with 25,842 edges and 1,375 nodes with 27,370 edges forming a connected network for winter and autumn, respectively.

Among the KOs specific to the autumn network, functions in the metabolic pathways for porphyrin and chlorophyll metabolism, sesquiterpenoid, triterpenoid and carotenoid biosynthesis pathways (ko00860, ko00909 and ko00906) were found to be enriched. This reinforces the notion that photosynthesis occurs in the OMMC sampled in autumn, while photosynthetic gene appear to be below the detection limit in the winter sample.

\section{Identification of season-specific metabolic traits}

The autumn- and winter-specific community-wide metabolic network reconstructions exhibit similar structures (Figure $3 \mathrm{f}, \mathrm{g}$ ) and represent 1,605 common KOs (i.e., 88 or $94 \%$ of the KOs included in the autumn or winter network reconstructions, respectively). Based on the reconstructed networks, a detailed network topological analysis was carried out (Supplementary Dataset 7).

Load scores (Equation 1) were determined in the reconstructed season-specific community-wide metabolic networks (Materials and methods). Most of the nodes in both the autumn- and winterspecific networks, which feature a high degree, represent KOs involved in amino acid synthesis. The relative small average shortest path lengths of 3.21 and 3.29 in the autumn and winter network reconstructions demonstrate that these represent 'small world' networks. ${ }^{61}$ Among the nodes with the highest betweenness centrality, i.e., the highest number of shortest paths passing through a node, ${ }^{62}$ in both metabolic reconstructions, KOs with functions in pyruvate metabolism, glycolysis or gluconeogenesis and glycerolipid metabolism were enriched (false discovery rateadjusted $P$ value $<0.05)$. In contrast, relatively higher betweenness centrality of the nodes representing KOs in fatty acid metabolism pathway (ko01212) was observed in the network reconstruction from the winter data set (median fold change of 4; Wilcoxon signed rank test $P$ value $<0.001$; enriched with false discovery rate-adjusted $P$ value $<0.00001$; Supplementary Figure 6, Supplementary Dataset 7) suggesting distinct substrate usage in both seasons. Other functions, in which this subset of KOs was enriched, included porphyrin and chlorophyll metabolism, biotin metabolism, polyketide sugar unit biosynthesis, lipoic acid metabolism and fluorobenzoate degradation (ko00860, ko00780, ko00523, ko00785 and ko00364), while only phosphoinositol metabolism (ko00562) was significantly enriched among the functions of the nodes with a higher betweenness centrality in the autumn network.

\section{Identification of genes encoding key functionalities}

Keystone species occupy topologically important positions in species interaction networks ${ }^{63}$ and are characterized by a high relative activity. ${ }^{17}$ Within a community-wide metabolic network reconstruction, key functionalities contributed by keystone populations should be encoded by genes which exhibit a high relative gene expression and these genes should also occupy important topological positions in relation to the community-wide metabolic network, i.e., they should represent 'load points'19 (Figure 1b). Herein, we therefore identify genes having a high load score (Equation 1) within the season-specific metabolic networks as well as high relative expression in the respective data sets (Figure 2b, Figure 4, Materials and methods). Selected genes are reported and potential 'choke points' are indicated in Supplementary Dataset 7. According to Rahman and Schomburg, choke points are special cases of load points, which consume and/or produce unique metabolites. Given that uniqueness of a metabolite is a strong claim in the context of the reconstructed community-wide metabolic networks as much of community metabolism remains unknown (only $13 \%$ of the predicted genes could be confidently annotated with a function), the identification of key functionalities by using load points was chosen as a more robust and appropriate measure in the present case. The positions of the key functionalities within the networks as per our criteria (Figure $1 \mathrm{~b}$ ) are indicated in Figure 4 and Supplementary Figure 7. KOs involved in porphyrin and chlorophyll metabolic pathways are enriched among the selected genes in the autumn community, as are KOs with a function in degradation of aromatic compounds. Among the genes encoding key functionalities in the winter OMMCs, no significant enrichment among KOs from a particular pathway could be observed. However, one of these genes is K03921, coding for an acyl-[acyl-carrier-protein] desaturase, which is part of the biosynthesis pathway for polyunsaturated fatty acids.

In both the autumn and winter sets of season-specific key genes, the subunits of ammonia or methane monooxygenase (AMO or MMO) stand out. As discussed above and given the sampling from a nitrifying-denitrifying wastewater treatment plant, this is likely an AMO which catalyses the first essential step of nitrification by converting ammonia to hydroxylamine. ${ }^{64}$ In contrast, MMO is involved in methane oxidation, which is less likely to be expressed in the sampled environment.

\section{Linking genes encoding key functionalities to community members}

Having selected genes encoding key functionalities within the sampled OMMCs using the reconstructed community-wide metabolic networks (Supplementary Dataset 7), we were interested in revealing which organisms expressed these genes within the community. As these genes contribute essential functionalities to the community and are characterized by relatively high expression, they are likely to be encoded by keystone species. Contigs containing genes annotated with one of the genes encoding key functionalities were selected from the combined metagenomic and metatranscriptomic data sets. These contigs were aligned to the NCBInr nucleotide database (Supplementary Dataset 7) to identify organisms encoding genes with similarity to the expressed genes of interest.

For five such genes (K03921, K01186, K01576, K01709 and K03335), no significant matches could be identified. On the other 
a

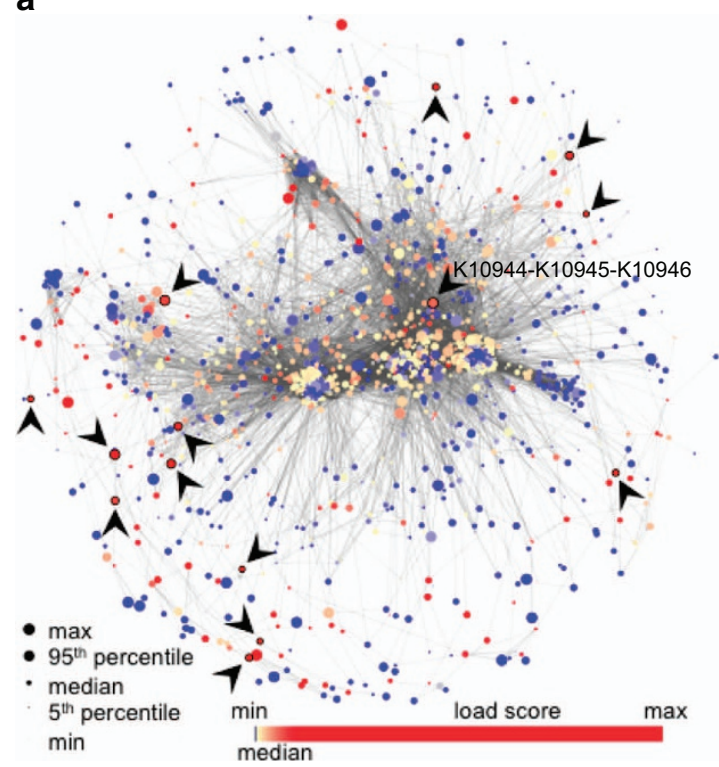

C

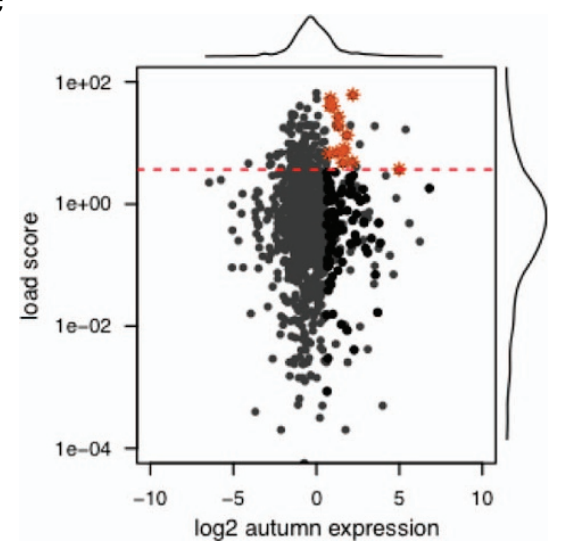

b

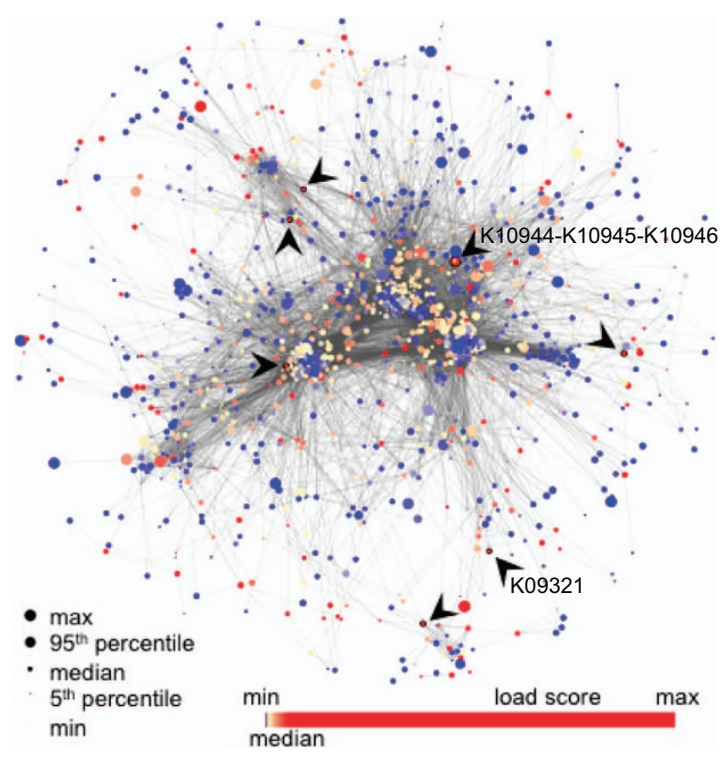

d

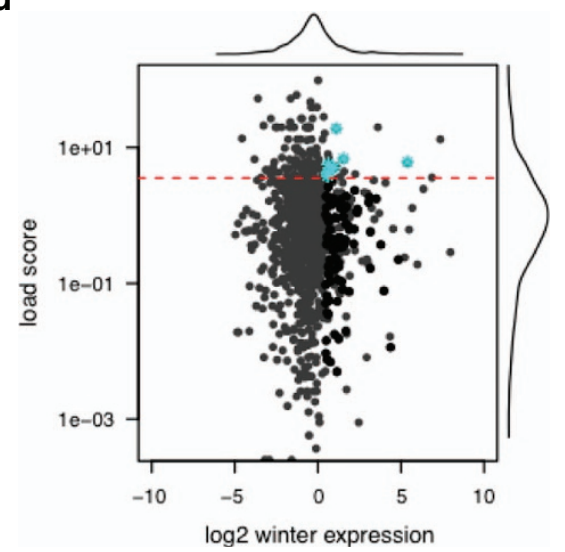

Figure 4. Topological analysis of the reconstructed season-specific community-wide metabolic networks and assessment of relative gene expression. (a) Autumn- and (b) winter-specific networks. In (a) and (b) node colours refer to load score and node sizes represent relative gene expression. KOs encoding key functionalities are encircled and highlighted by arrow heads. (c and d) Results of the topological analysis of KOs in simplified season-specific networks for (c) autumn and (d) winter. Highly expressed genes are indicated as black dots and KOs encoding key functionalities are indicated by brown (autumn) or cyan (winter) asterisks. Dotted red lines indicate minimal load score of KOs deemed to encode key functionalities.

hand, three of these key genes from the winter-specific network (K01251, K00789 and K03527) were expressed from a multitude of contigs, which could be aligned well to over 50 different species. Half of the matched contigs encoding the five autumn key genes from the chlorophyll- and porphyrin-synthesis pathway (K03403, K03404, K03405, K04034, K04035) were most similar to sequences encoded by the genome of the cyanobacterium Oscillatoria nigro-viridis PCC 712. The relative expression of these genes accounted for $85 \%$ of the expression of these genes in autumn (Supplementary Dataset 7). Some Oscillatoria spp. are found in wastewater, where they have been found to participate in nitrate removal. ${ }^{65}$

From the list of genes encoding key functionalities, we further selected the acyl-[acyl-carrier protein] desaturase (K03921) and the three subunits of AMO or MMO (K10944, K10945 and K10946) for further analysis. In all, 922 out of 1,067 contigs belonging to the AMO or MMO complex matched best to sequences of Nitrosomonas spp. a well-known genus of nitrifiers. The other contigs matched sequences from uncultured organisms or, in two cases, to a MMO from Methylovulum miyakonense. These two cases only represented $0.1 \%$ of the total contig length of the KOs K10944-K10946. Furthermore, less than 1\% of the metatranscriptomic reads mapped to these two contigs, suggesting that the major function of these $\mathrm{KOs}$ is in ammonia rather than methane oxidation. In addition, a refined assembly of contigs belonging to K10944-K10946 using additional metagenomic data from a third sampling date (Supplementary Materials and methods) yielded a new contig containing complete sequences for $a m o A$ (an established phylogenetic marker for nitrifying microorganisms ${ }^{66}$ ), and $a m o B$, both also matching best to Nitrosomonas spp. A phylogenetic tree was reconstructed using the predicted amino acid sequence of AmoA from this contig and the tree clearly places it closest to sequences of Nitrosomonas spp. (Figure 5a, Supplementary Table 4). To estimate the abundance of Nitrosomonas spp. in the sampled OMMCs, metagenomic and metatranscriptomic reads were mapped against the genome 

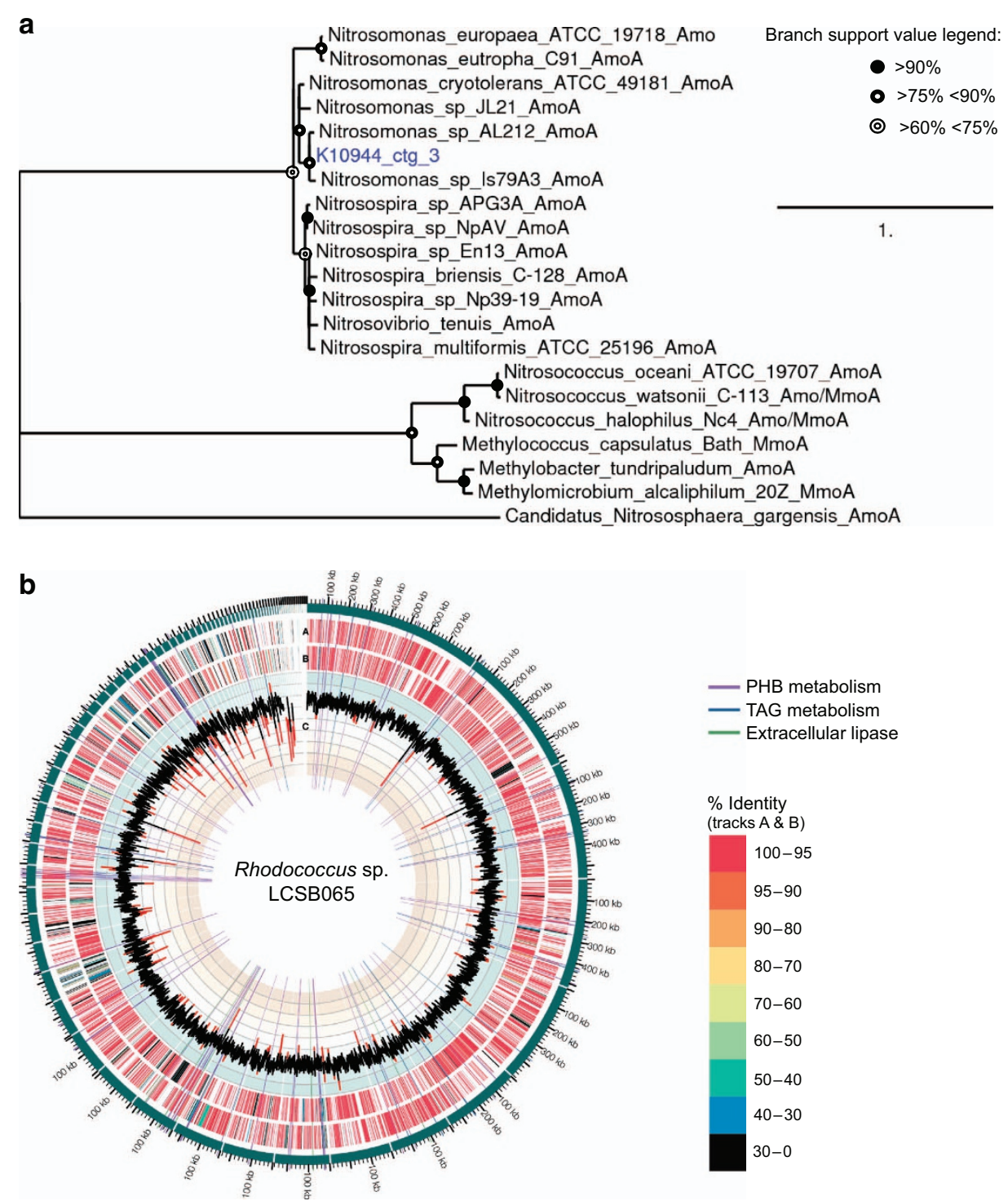

Figure 5. Linking key functionalities to important community members. (a) Phylogenetic tree based on the AmoA amino acid sequence derived from a contig extended using combined metagenomic and metatranscriptomic data (K10944_ctg_3). (b) Circos plot of the genome of Isolate LCSB065, highlighting amino acid similarity of encoded proteins to the Rhodococcus erythropolis PR4 genome and genes involved in poly-hydroxybutyrate (PHB) and TAG accumulation as well as encoded extracellular lipases. From the outside to the inside track: contigs (green) arranged by size; A: open reading frames in forward direction; B: open reading frames in reverse direction; colours in tracks $\mathrm{A}$ and $\mathrm{B}$ indicate \%-similarity to the Rhodococcus erythropolis PR4 genome; C: $\% \mathrm{G}+\mathrm{C}$ in 1,000 bp sliding windows. Highlighted rays indicate the location of genes involved in PHB metabolism (violet), genes involved in TAG metabolism (blue) and extracellular lipase genes (green). TAG, triacylglycerol.

sequence of Nitrosomonas sp. Is79 (ref. 67), yielding approximately twice as many metagenomic reads in winter compared with autumn (Supplementary Table 5). The ratio of metatranscriptomic to metagenomic coverage was four times higher in winter than in autumn, indicating a higher general level of activity of Nitrosomonas spp. in the winter OMMC, although AMO activity was high in both seasons.

In contrast to the compelling link between the putative AMO genes and Nitrosomonas spp., linking the acyl-[acyl-carrier protein] desaturase unambiguously to an organismal group could not be achieved by simple alignment to reference genomes in public databases. Of the 14 contigs which harboured genes annotated with K03921 expressed in the winter sample, 9 did not yield any hits with a percentage identity $>80 \%$ and query coverage $>50 \%$. The remaining five contigs yielded hits with 82 to $86 \%$ identity to sequences from Rhodococcus erythropolis, Amycolatopsis mediterranei and Nocardia cyriacigeorgica. As none of these alignments were of high confidence, we aligned the contigs encoding acyl-[acylcarrier protein] desaturases to genomes of an in-house bacterial isolate collection from the same BWWT plant. Three of the contigs containing expressed genes matched to the same gene of the genome of Isolate LCSB065 with 88 to $100 \%$ identity over a total of $459 \mathrm{nt}$ of the combined metagenomic contig length of $678 \mathrm{nt}$. Isolate LCSB065's 81 contigs contain an almost complete $7.67 \mathrm{Mbp}$ genome with a GC-content of $62.4 \%$ (Figure 5b, Supplementary Dataset 8). Based on the use of 31 bacterial protein coding marker genes, this isolate was identified as a Rhodococcus sp. ${ }^{68}$ (Supplementary Dataset 8). A detailed genomic analysis revealed a high number of genes involved in lipid metabolism encoded by this organism (Supplementary Results and Discussion) and non-polar 
storage granules were also observed microscopically (Supplementary Figure 8). As Rhodococcus spp. are known to exhibit lipid accumulation phenotypes, ${ }^{69}$ it is likely that this organism is a keystone species within the OMMC. Recruitment of metagenomic and metatranscriptomic reads to the isolate's genome (Supplementary Dataset 8) revealed a low abundance of this organismal group in both autumn and winter, with a relative high transcriptional activity only in winter (Figure 5b, Supplementary Table 5) potentially directly linking its activity to the high community-wide lipid accumulation phenotype observed in winter. ${ }^{24}$ Low abundance combined with an activity with a great impact on their environment are hallmarks of keystone species and the Rhodococcus population fulfils these criteria in the context of the sampled OMMC.

\section{CONCLUSION}

Despite stark differences in the appearance and structure of the sampled autumn and winter OMMCs, the comparative analysis of integrated metagenomic, metatranscriptomic and metaproteomic data contextualised in reconstructed community-wide metabolic networks uncovered surprisingly few global differences in terms of functional genetic potential and gene expression between the two communities. This result confirms previous observations that taxonomic profiles can be very variable whereas global functional profiles are typically more conserved. ${ }^{70,71}$ Nonetheless, our approach highlighted genes coding for essential enzymes involved in nitrogen metabolism (genes involved in nitrification, denitrification and ammonium oxidation) as being relatively highly expressed in both seasons despite exhibiting only low gene copy numbers. Identified differences between the two seasons include a marked expression of enzymes involved in glycerolipid metabolism in winter when OMMC biomass is most pronounced (Figure 2a,b) and lipid accumulation is higher. ${ }^{24}$ In particular, our analyses highlight the importance of triacylglycerol lipases, which are essential for hydrolysis of lipids into long-chain fatty acids and their subsequent assimilation and intracellular storage. The pronounced expression of this particular enzyme group suggests the possibility to enrich for lipid accumulating organisms (LAOs) in BWWT plants through lipase supplementation and environmental biocatalysis. Enhancing the growth of LAOs through such a strategy would result in the availability of excess amounts of lipid-rich biomass at the air-water interface of anoxic tanks and this could, for example, be transesterified to biodiesel, thereby allowing recovery of a significant fraction of the chemical energy contained within wastewater. ${ }^{28,29}$

The topological analysis of the OMMC-wide metabolic networks confirms the metabolic similarity of both autumn and winter communities, with a high centrality of central carbon metabolism. The measure of betweenness centrality demonstrates seasonal variability in fatty acid metabolism, which is more enriched in the sampled winter OMMC. The identification of genes encoding key functionalities involved the detailed analysis of topological features within the reconstructed community-wide metabolic networks as well as an assessment of relative gene expression by enzyme-coding genes. This analysis highlighted genes such as AMO, expressed by Nitrosomonas spp., and an acyl-[acyl-carrier protein] desaturase, expressed by Rhodococcus spp., as fulfilling key functions in OMMCs.

The developed framework allows the integration of structural and functional measurements through contextualisation in reconstructed community-wide metabolic networks to result in the identification of genes encoding key functionalities, which can in turn be linked to functionally important community members. These potential 'keystone genes' could ultimately serve as driver nodes $^{31}$ for controlling such complex microbial ecosystems. Therefore, the application of our methodological framework to other microbial communities for the identification of keystone species may allow community-wide control strategies to be formulated where other community-wide phenotypic outcomes may be desirable, e.g., in the human gastrointestinal tract. In silico analysis results presented in this paper were obtained using the high performance computing facilities of the University of Luxembourg ${ }^{51}$.

\section{ACKNOWLEDGEMENTS}

The authors thank Mr Bissen and Mr Di Pentima from the Syndicat Intercommunal à Vocation Ecologique (SIVEC) for their permission to collect samples and gain access to the monitoring platform of the Schifflange wastewater treatment plant; Dr Nicolás Pinel for his refinement of genome assembly and visualisation of Rhodococcus sp. LCSB065 and helpful discussions; and Dr Aidos Baumuratov for his support for microscopic analyses of the OMMC isolates. This work was supported by an ATTRACT programme grant (ATTRACT/A09/03) and a European Union Joint Programming in Neurodegenerative Diseases grant (INTER/JPND/12/01) both to PW, and Aide à la Formation Recherche (AFR) grants to HR (PHD-MARP-04), EELM (PRD-2011-1/SR), CCL (PHD/4964712) and SN (PHD-2014-1/7934898), all funded by the Luxembourg National Research Fund (FNR). The project received financial support from the Integrated Biobank of Luxembourg with funds from the Luxembourg Ministry of Higher Education and Research. The work of PM was funded by the 'Plan Technologies de la Santé du Gouvernement du Grand-Duché de Luxembourg' through the Luxembourg Centre for Systems Biomedicine (LCSB), University of Luxembourg. This work was also supported in part with US federal funds from the National Science Foundation MRI grant No. 0923536, by funds through National Institutes of Health; from the National Institute of General Medical Sciences under grant No. 2P50 GM076547/Centre for Systems Biology and S10RR027584.

\section{CONTRIBUTIONS}

$\mathrm{HR}, \mathrm{AH}-\mathrm{B}$ and PW designed the study; HR, EELM, LAL and PW sampled the treatment plant and extracted biomolecules; AH-B, HR, PM, VPS, CCL, SN, JMS, JDG, NDH, DME and PSK analysed the metagenomic and metatranscriptomic data; $M R H, A H-B, H R$, PM, RLM and VPS analysed the metaproteomic data; HR, AH-B, TS and VPS reconstructed and analysed the metabolic networks; $A H-B, H R$ and PW wrote the manuscript. All the authors discussed the results and commented on the manuscript.

\section{COMPETING INTERESTS}

The authors declare no conflict of interest.

\section{REFERENCES}

1 Muller EE, Glaab E, May P, Vlassis N, Wilmes P. Condensing the omics fog of microbial communities. Trends Microbiol 2013; 7: 325-333.

2 Helbling DE, Ackermann M, Fenner K, Kohler H-PE, Johnson DR. The activity level of a microbial community function can be predicted from its metatranscriptome. ISME J 2012; 6: 902-904.

3 Wilmes P, Bond PL. Microbial community proteomics: elucidating the catalysts and metabolic mechanisms that drive the Earth's biogeochemical cycles. Curr Opin Microbiol 2009; 12: 310-317.

4 Roume H, Muller EE, Cordes T, Renaut J, Hiller K, Wilmes P. A biomolecular isolation framework for eco-systems biology. ISME J 2013; 7: 110-121.

5 Tang J. Microbial metabolomics. Curr Genomics 2011; 12: 391-403.

6 Oberhardt MA, Palsson BO, Papin JA. Applications of genome-scale metabolic reconstructions. Mol Syst Biol 2009; 5: 320.

7 Cottret L, Milreu PV, Acuña V, Marchetti-Spaccamela A, Stougie L, Charles H et al. Graph-based analysis of the metabolic exchanges between two co-resident intracellular symbionts, Baumannia cicadellinicola and Sulcia muelleri, with their insect host, Homalodisca coagulata. PLoS Comput Biol 2010; 6: e1000904.

8 Wintermute $\mathrm{EH}$, Silver PA. Emergent cooperation in microbial metabolism. Mol Syst Biol 2010; 6: 407.

9 Greenblum S, Chiu H-C, Levy R, Carr R, Borenstein E. Towards a predictive systems-level model of the human microbiome: progress, challenges, and opportunities. Curr Opin Biotech 2013; 24: 810-820.

10 Abubucker S, Segata N, Goll J, Schubert AM, Izard J, Cantarel BL et al. Metabolic reconstruction for metagenomic data and its application to the human microbiome. PLoS Comput Biol 2012; 8: e1002358.

11 Konwar KM, Hanson NW, Pagé AP, Hallam SJ. MetaPathways: a modular pipeline for constructing pathway/genome databases from environmental sequence information. BMC Bioinformatics 2013; 14: 202. 
12 Greenblum S, Turnbaugh PJ, Borenstein E. Metagenomic systems biology of the human gut microbiome reveals topological shifts associated with obesity and inflammatory bowel disease. Proc Natl Acad Sci USA 2012; 109: 594-599.

13 Borenstein E. Computational systems biology and in silico modeling of the human microbiome. Brief Bioinform 2012; 13: 769-780

14 Steele JA, Countway PD, Xia L, Vigil PD, Beman JM, Kim DY et al. Marine bacterial, archaeal and protistan association networks reveal ecological linkages. ISME J 2011; 5: 1414-1425.

15 Berry $\mathrm{D}$, Widder S. Deciphering microbial interactions and detecting keystone species with co-occurrence networks. Front Microbiol 2014; 5: 219.

16 Paine RT. A conversation on refining the concept of keystone species. Conserv Biol 1995; 9: 962-964.

17 de Visser S, Thébault E, de Ruiter PC. Ecosystem Engineers, Keystone Species. In: Leemans R (ed). Ecological Systems. Springer: New York, NY, USA, 2013; 59-68.

18 Ze X, Duncan SH, Louis P, Flint HJ. Ruminococcus bromii is a keystone species for the degradation of resistant starch in the human colon. ISME J 2012; 6 : $1535-1543$

19 Rahman SA, Schomburg D. Observing local and global properties of metabolic pathways:'load points' and 'choke points' in the metabolic networks. Bioinformatics 2006; 22: 1767-1774.

20 Daims H, Taylor MW, Wagner M. Wastewater treatment: a model system for microbial ecology. Trends Biotechnol 2006; 24: 483-489.

21 Denef VJ, Mueller RS, Banfield JF. AMD biofilms: using model communities to study microbial evolution and ecological complexity in nature. ISME J 2010; 4 599-610.

22 Eckburg PB, Bik EM, Bernstein CN, Purdom E, Dethlefsen L, Sargent M et al. Diversity of the human intestinal microbial flora. Science 2005; 308: 1635-1638.

23 Mocali S, Benedetti A. Exploring research frontiers in microbiology: the challenge of metagenomics in soil microbiology. Res Microbiol 2010; 161: 497-505.

24 Muller EE, Pinel N, Laczny CC, Hoopmann MR, Narayanasamy S, Lebrun LA et al. Community-integrated omics links dominance of a microbial generalist to fine-tuned resource usage. Nat Commun 2014; 5: 1-10.

25 Chao A. Estimating the population size for capture-recapture data with unequal catchability. Biometrics 1987; 43: 783-791.

26 Zhang T, Shao M-F, Ye L. 454 Pyrosequencing reveals bacterial diversity of activated sludge from 14 sewage treatment plants. ISME J 2012; 6: 1137-1147.

27 Roume $\mathrm{H}$, Heintz-Buschart A, Muller EE, Wilmes P. Sequential isolation of metabolites, RNA, DNA, and proteins from the same unique sample. Microbia Metagenomics, Metatranscriptomics, and Metaproteomics. Method Enzymol 2013 531: 219-236.

28 Sheik AR, Muller E, Wilmes P. A hundred years of activated sludge: time for a rethink. Front Microbiol 2014; 5: 47.

29 Muller EE, Sheik AR, Wilmes P. Lipid-based biofuel production from wastewater. Curr Opin Biotechnol 2014; 30: 9-16.

30 Narayanasamy S, Muller EEL, Sheik AR, Wilmes P. Integrated omics for the identification of key functionalities in biological wastewater treatment microbial communities. Microb Biotechnol 2015; 8: 363-368.

31 Liu Y-Y, Slotine J-J, Barabási A-L. Controllability of complex networks. Nature 2011; 473: $167-173$.

32 Kofler R, Orozco-terWengel P, De Maio N, Pandey RV, Nolte V, Futschik A et al. PoPoolation: a toolbox for population genetic analysis of next generation sequencing data from pooled individuals. PLoS One 2011; 6: e15925.

33 Masella AP, Bartram AK, Truszkowski JM, Brown DG, Neufeld JD. PANDAseq: paired-end assembler for illumina sequences. BMC Bioinformatics 2012; 13: 31

34 Kultima JR, Sunagawa S, Li J, Chen W, Chen H, Mende DR et al. MOCAT: a metagenomics assembly and gene prediction toolkit. PLOS ONE 2012; 7: e47656.

35 Hyatt D, Chen G-L, LoCascio PF, Land ML, Larimer FW, Hauser LJ. Prodigal: prokaryotic gene recognition and translation initiation site identification. $B M C$ Bioinformatics 2010; 11: 119.

36 Rho $\mathrm{M}$, Tang $\mathrm{H}$, Ye $\mathrm{Y}$. FragGeneScan: predicting genes in short and error-prone reads. Nucleic Acids Res 2010; 38: e191.

37 Fu L, Niu B, Zhu Z, Wu S, Li W. CD-HIT: accelerated for clustering the nextgeneration sequencing data. Bioinformatics 2012; 28: 3150-3152.

38 Meyer F, Paarmann D, D'Souza M, Olson R, Glass EM, Kubal M et al. The metagenomics RAST server-a public resource for the automatic phylogenetic and functional analysis of metagenomes. BMC Bioinformatics 2008; 9: 386.

39 Kanehisa M, Goto S, Sato Y, Kawashima M, Furumichi M, Tanabe M. Data, information, knowledge and principle: back to metabolism in KEGG. Nucleic Acids Res 2014; 42: D199-D205.

40 Lee S, Seo CH, Lim B, Yang JO, Oh J, Kim M et al. Accurate quantification of transcriptome from RNA-Seq data by effective length normalization. Nucleic Acids Res 2011; 39: e9.

41 Craig R, Cortens JP, Beavis RC. Open source system for analyzing, validating, and storing protein identification data. J Proteome Res 2004; 3: 1234-1242.
42 Deutsch EW, Mendoza L, Shteynberg D, Farrah T, Lam H, Tasman N et al. A guided tour of the trans-proteomic pipeline. Proteomics 2010; 10: 1150-1159.

43 Keller A, Nesvizhskii Al, Kolker E, Aebersold R. Empirical statistical model to estimate the accuracy of peptide identifications made by MS/MS and database search. Anal Chem 2002; 74: 5383-5392.

44 Shteynberg D, Deutsch EW, Lam H, Eng JK, Sun Z, Tasman N et al. iProphet: multi-level integrative analysis of shotgun proteomic data improves peptide and protein identification rates and error estimates. Mol Cell Proteomics 2011; 10: M111,007690.

45 Kent WJ. BLAT - the BLAST-like alignment tool. Genome Res 2002; 12: 656-664.

46 Faust K, Croes D, van Helden J. Metabolic pathfinding using RPAIR annotation. J Mol Biol 2009; 388: 390-414.

47 Smoot ME, Ono K, Ruscheinski J, Wang P-L, Ideker T. Cytoscape 2.8: new features for data integration and network visualization. Bioinformatics 2011; 27: 431-432.

48 Fowler SD, Greenspan P. Application of Nile red, a fluorescent hydrophobic probe for the detection of neutral lipid deposits in tissue sections: comparison with oil red O. J Histochem Cytochem 1985; 33: 833-836.

49 Simpson JT, Wong K, Jackman SD, Schein JE, Jones SJ, Birol İ. ABySS: a paralle assembler for short read sequence data. Genome Res 2009; 19: 1117-1123.

50 Bankevich A, Nurk S, Antipov D, Gurevich AA, Dvorkin M, Kulikov AS et al. SPAdes: a new genome assembly algorithm and its applications to single-cell sequencing. J Comput Biol 2012; 19: 455-477.

51 Varrette S, Bouvry P, Cartiaux H, Georgatos F. Management of an Academic HPC Cluster: The UL Experience. Proceedings of the 2014 International Conference on High Performance Computing Simulation (HPCS 2014). IEEE: Bologna, Italy, 2014.

52 Hettich RL, Sharma R, Chourey K, Giannone RJ. Microbial metaproteomics: identifying the repertoire of proteins that microorganisms use to compete and cooperate in complex environmental communities. Curr Opin Microbiol 2012; 15: 373-380.

53 Carvalhais LC, Dennis PG, Tyson GW, Schenk PM, de Bruijn F. Rhizosphere metatranscriptomics: challenges and opportunities. de Bruijn FJ (ed). Molecula Microbiology of the Rhizosphere. Wiley-Blackwell: New Jersey, NJ, USA, 2013 1137-1144.

54 Tsementzi D, Poretsky R, Rodriguez-R LM, Luo C, Konstantinidis KT. Evaluation of metatranscriptomic protocols and application to the study of freshwater microbial communities. Environ Microbiol Rep 2014; 6: 640-655.

55 Zhu G, Peng Y, Li B, Guo J, Yang Q, Wang S. Biological removal of nitrogen from wastewater. In: Reviews of Environmental Contamination and Toxicology. Springer: New York, NY, USA, 2008; 159-195.

$56 \mathrm{Yu} \mathrm{K}$, Zhang T. Metagenomic and metatranscriptomic analysis of microbial community structure and gene expression of activated sludge. PLoS ONE 2012; 7 e38183.

57 Kalscheuer R. Genetics of wax ester and triacylglycerol biosynthesis in bacteria. In: Timmis KN (ed). Handbook of Hydrocarbon and Lipid Microbiology. Springer: Berlin Heidelberg, Germany, 2010; 527-535.

58 Muller EEL, Pinel N, Gillece JD, Schupp JM, Price LB, Engelthaler DM et al. Genome Sequence of 'Candidatus Microthrix parvicella' Bio17-1, a long-chain-fatty-acidaccumulating filamentous actinobacterium from a biological wastewater treatment plant. J Bacteriol 2012; 194: 6670-6671.

59 Mcllroy SJ, Kristiansen R, Albertsen M, Karst SM, Rossetti S, Nielsen JL et al. Metabolic model for the filamentous 'Candidatus Microthrix parvicella' based on genomic and metagenomic analyses. ISME J 2013; 7: 1161-1172.

60 De Clippeleir H, Vlaeminck SE, De Wilde F, Daeninck K, Mosquera M, Boeckx P et al. One-stage partial nitritation/anammox at $15{ }^{\circ} \mathrm{C}$ on pretreated sewage: feasibility demonstration at lab-scale. App Microbiol Biotechnol 2013; 97: 10199-10210.

61 Raunkjær K, Hvitved-Jacobsen T, Nielsen PH. Measurement of pools of protein, carbohydrate and lipid in domestic wastewater. Water Res 1994; 28 : 251-262.

62 Watts DJ, Strogatz SH. Collective dynamics of 'small-world'networks. Nature 1998; 393: 440-442.

63 Brandes U. On variants of shortest-path betweenness centrality and their generic computation. Soc Networks 2008; 30: 136-145.

64 Faust K, Raes J. Microbial interactions: from networks to models. Nat Rev Microbiol 2012; 10: 538-550

65 Martens-Habbena W, Berube PM, Urakawa H, José R, Stahl DA. Ammonia oxidation kinetics determine niche separation of nitrifying Archaea and Bacteria. Nature 2009; 461: 976-979.

66 Attasat S, Wanichpongpan P, Ruenglertpanyakul W. Cultivation of microalgae (Oscillatoria okeni and Chlorella vulgaris) using tilapia-pond effluent and a comparison of their biomass removal efficiency. Water Sci Technol 2013; 67: 271-277.

67 Liu W, Li L, Khan MA, Zhu F. Popular molecular markers in bacteria. Mol Genet Microbiol Virol 2012; 27: 103-107. 
68 Bollmann A, Sedlacek CJ, Norton J, Laanbroek HJ, Suwa Y, Stein LY et al. Complete genome sequence of Nitrosomonas sp. Is79, an ammonia oxidizing bacterium adapted to low ammonium concentrations. Stand Genomic Sci 2013; 7: 469.

69 Kerepesi C, Bánky D, Grolmusz V. AmphoraNet: the webserver implementation of the AMPHORA2 metagenomic workflow suite. Gene 2014; 533: 538-540.

70 Alvarez HM, Mayer F, Fabritius D, Steinbüchel A. Formation of intracytoplasmic lipid inclusions by Rhodococcus opacus strain PD630. Arch Microbiol 1996; 165: 377-386.

71 Ju F, Guo F, Ye L, Xia Y, Zhang T. Metagenomic analysis on seasonal microbial variations of activated sludge from a full-scale wastewater treatment plant over 4 years. Environ Microbiol Rep 2014; 6: 80-89.
72 Xu Z, Malmer D, Langille MGl, Way SF, Knight R. Which is more important for classifying microbial communities: who's there or what they can do? ISME J 2014; 8: 2357-2359.

(c) (i)

This work is licensed under a Creative Commons Attribution 4.0 International License. The images or other third party material in this article are included in the article's Creative Commons license, unless indicated otherwise in the credit line; if the material is not included under the Creative Commons license, users will need to obtain permission from the license holder to reproduce the material. To view a copy of this license, visit http://creativecommons.org/licenses/ by/4.0/

Supplementary Information accompanies the paper on the npj Biofilms and Microbiomes website (http://www.nature.com/npjbiofilms) 